\title{
Bullet Train Physics (with) Gill's Electronic Theory of Magnetism (1964)
}

\author{
Avtar Singh Gill, MD \\ avtargillmd@aol.com
}

\begin{abstract}
A summarized Gill's electronic theory of magnetism (1964) shows diagrammatically and experimentally that a magnet has a negative magnetic pole and a positive magnetic pole. These two magnetic poles are also called the north and south magnetic poles owing to the direction of a magnetic compass on the surface of the Earth. The Tesla unit is explained with the help of Gill's electronic theory of magnetism (1964) combined with Coulomb's law (1784).

An economical L-shaped electro-magnet will be used under the 'Bullet Train' and above the rail track. The rail track will also conduct a direct electric current on its surface.

The exposed electron dependent negative magnetic pole under and at the train wheel level is repelled by the electrons flowing as a direct electric current on the surface of the rail track. This levitation of the 'Bullet train' results in loss of resistance between the train wheels and the rail track. Dot-product calculations will be offered for this levitation.
\end{abstract}

The levitated train will be pulled longitudinally with the linear motor in front of the train which has the exposed proton dependent positive magnetic pole. The negative electron dependent direct electric current flow on the railway track results in attractional force between the proton (+e)dependent positive magnetic pole on the front of the train and the flowing electrons (-e) in the metallic strip on the surface of the railway track. While the 'Bullet train' is levitated and has minimal friction', this longitudinal attractional force results in great speed. This will also be presented as a dot-product calculation.

The linear motor on the front of the train is a flexible positive electro-magnetic pole of the electro-magnet which will help in varying the speed and direction of the 'Bullet Train'.

A brief discussion will follow offering the economical and mathematical advantages of the above 'Bullet Train' over the existing 'Magelev system'.

'Gill's electronic theory of magnetism 1964'also shows that there is no asymmetry between the electrical and magnetic forces.

\section{INTRODUCTION}

Gill's electronic theory of magnetism (1964) resolves the asymmetry between magnetic and electrical forces which was because of 'Maxwell's dipole theory of magnetism (1873)'.

In this article, the author will justify the above statement by showing that if we apply 'Gill's electronic theory of magnetism (1964)'instead of 'Maxwell's dipole theory of magnetism (1873)', we come up with a simple design of the 'Bullet Train' with dot product calculations.

According to Gill's electronic theory of magnetism (1964), a magnet has an exposed electron dependant negative magnetic pole at one end and an exposed proton dependant positive magnetic pole at the other end. As these two positive and negative magnetic poles of a magnetic compass are pointed towards the north and south poles of the magnetic Earth, they are commonly called the north magnetic pole and the south magnetic pole of the magnet.

An economical L-shaped electro-magnet will be used and the interaction of its negative and positive magnetic poles with the electron based direct electric current force in the metallic strip on the rail track will help with the levitation and the linear motor for longitudinal movement respectively of the 'Bullet Train'.

The levitation of the 'Bullet Train' is caused by repulsion between the negative force from the exposed inner electron $(-e)$ dependent negative(north) magnetic pole at/under the wheels of the train and the negative electrical force $(-e)$ on the surface of the rail track as a direct electric current. Dot product equations to support the levitation will be offered. On the front of the 'Bullet Train', the exposed proton $(+e)$ dependent positive (south) magnetic pole in front of the train acts as a flexible linear motor. This is attracted by the direct negative electrical current force $(-e)$ on the surface of the rail track and causes 
longitudinal movement of the train in the direction of the negative direct electric current flow on the rail track. Dot-product equations to support the same will be offered.

The known Maglev system of levitated 'Bullet Trains' uses two or more sets of magnets. One set of magnets to levitate the train off the track and the second set of magnets or winding coils to cause a linear motor type of magnetic induction effect to make the frictionless train move at great speed. The application of Gill's electronic theory of magnetism (1964) shows that no two sets of magnets for levitation and no two sets of magnets for linear motor function are required for the Bullet Train.

Gill's electronic theory of magnetism (1964) facilitates the usage of dot-product calculations unlike Maxwell's dipole theory of magnetism (1873) which forces us to apply the not so clear cross-products devised by Lorentz (1893).

\section{METHOD}

Gill's electronic theory of magnetism (1964) will be summarized. This helps to explain that the same inner electron and proton dependent forces which manifest at the two ends of a magnet as a negative (north) magnetic pole and a positive (south) magnetic pole are also responsible for an electric current with the rapid flow of the outer free valence electrons along a metallic conductor which in this case will be on the surface of the rail track. Coulomb's law (1784) combined with Gill's electronic theory of magnetism (1964) will explain the Tesla unit.

The flexible core of the electro-magnet on the train will be shaped like an L with its electron (-e) dependent negative magnetic (north) pole under the wheel facing the track. The rail track has a direct electric current on its surface flowing as electrons (-e).

The repulsion between the electron dependant (-e) negative magnetic pole and the electrons (-e) travelling on the surface of the rail track as a direct electric current results in levitation of the train. Dot product equations will be submitted for the levitation force.

On the front of the train pointed forward in the direction of the track is the linear motor as a flexible exposed proton (+e) dependent positive magnetic pole of the L-shaped electro-magnet. This will result in attraction of the levitated train in the direction of electron (-e) dependent negative direct electric current flow on the rail track. Dot product equations will be offered.

The change of direction of the flexible positive $(+\mathrm{e})$ electro-magnetic pole in front of the train from perpendicular downward towards the rail track $\left(T=90^{\circ}\right)$ to forward towards the rail track $\left(-=^{\circ}\right)$ will increase the speed of the levitated train from stand-still to maximum speed. If the direction of the positive magnetic poleis turned backwards towards the train to $\left(>90^{\circ}\right.$ eg $\left.120^{\circ}\right)$, this attractional force can be used to move the train backwards or to slow it down while levitated.

\section{Gill's EleCtronic Theory of MAGnetism (1964)}

Gill's electronic theory of magnetism (1964) is based on the structure of the atom and explains how the positively charged protons (+e) and the negatively charged electrons (-e) of an atom are responsible for both magnetism and the electrical forces.

In the diagrams that follow in this article, we are using a simplified version of the structure of an atom with large black proton mass and small red inner electrons. Please note that the outer free valence electrons (not shown) take part mainly in electrical current flow. Do note that non-moving charges do not manifest as an electric current but manifest as a magnetic force.

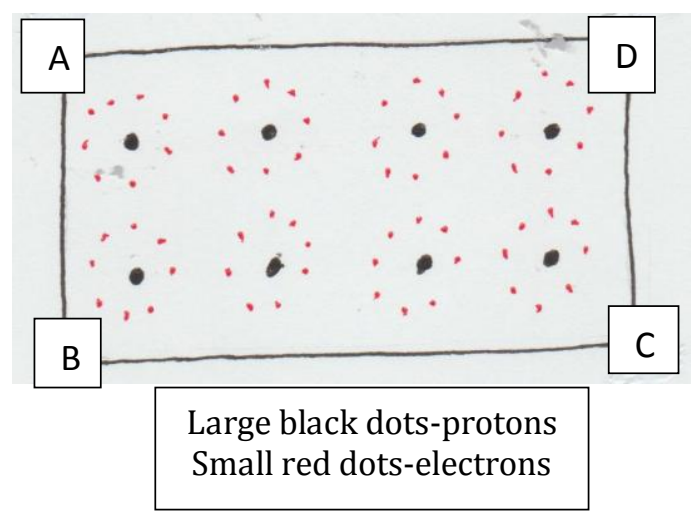

Figure1 (a). Neutral iron atoms in an un-magnetized state 


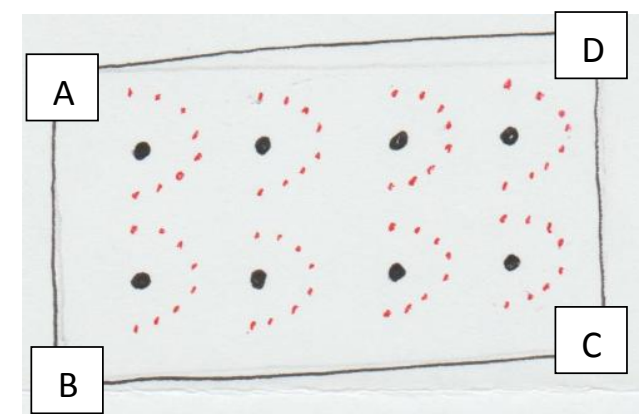

Figure1 (b). Change in position of the inner electrons on magnetization.

According to Gill's electronic theory of magnetism (1964), the neutral iron atoms in Fig1a are magnetized in Figure $1 \mathrm{~b}$ and $\mathrm{CD}$ has become the negative magnetic pole or north magnetic pole with a negatively torque $(-\boldsymbol{\tau})$ non-moving exposed inner electron based charge $-\boldsymbol{q}=\boldsymbol{n}(-\boldsymbol{e})=-\boldsymbol{n} \boldsymbol{e}$ coulombs of the magnet and $\mathrm{AB}$ has become the positive magnetic pole or the south magnetic pole of the magnet with an opposite positively torqued $(+\boldsymbol{\tau})$ non-moving charge $+\boldsymbol{q}=\boldsymbol{n}(+\boldsymbol{e})=+\boldsymbol{n} \boldsymbol{e}$ coulombs where $\boldsymbol{n}$ is the number of exposed inner electrons at one end and equals the number of exposed protons at the other end of the magnet.

The neutral atoms in Fig 1 a have become magnetized atoms in Fig $1 b$ by undergoing a change in configuration and each atom also has developed an opposing torque between its own electrons and protons to give the magnetized atoms a cork-screw effect.

Experiment- A physicist showed me the following experiment in 1965. On a wooden table, spread some coarse iron filings and in the middle of the iron filings, place a magnet

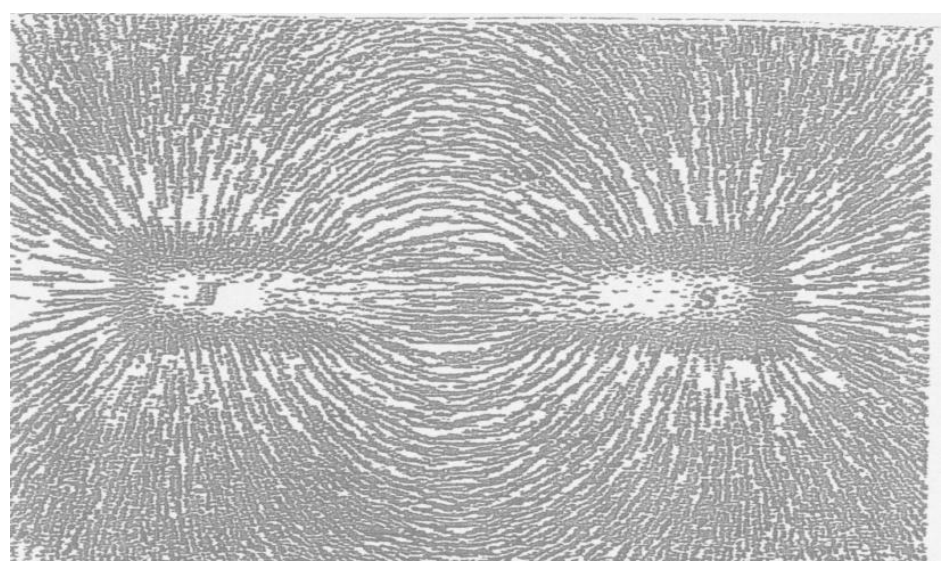

Figure2 (a). A bar magnet with iron filings arranged along magnetic field lines

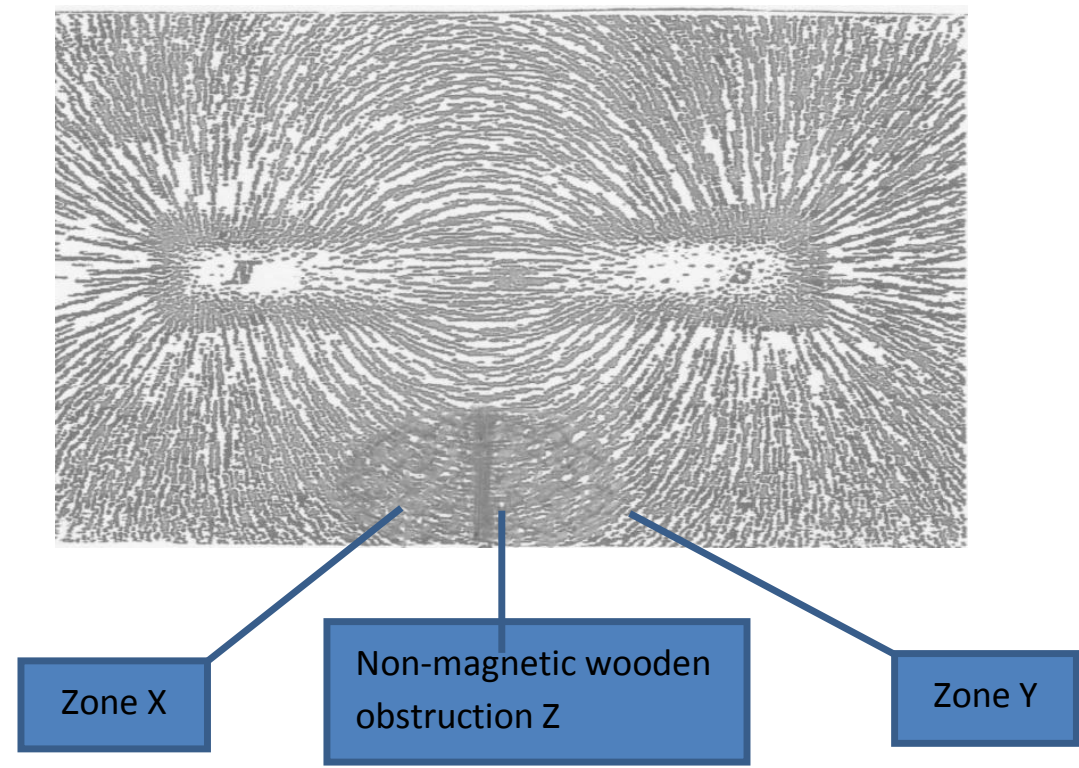

Figure2 (b). A wooden non-magnetic obstruction $Z$ is placed on one side on the iron filings 
In Fig $2 \mathrm{~b}$, a wooden non-magnetic obstruction $\mathrm{Z}$ is placed on one side on the iron filings. The iron filings crumple on both sides of $\mathrm{Z}$ in zones $\mathrm{X}$ and $\mathrm{Y}$. If the magnetic force was a single force, the iron filings should have crumpled in Zone X or Zone Y only.

Thus, the magnetic force is combination of the proton $(+e)$ based positive and electron $(-e)$ based negative forces from the two poles of a magnet seen both diagrammatically and experimentally.

According to Gill's electronic theory of magnetism (1964), the magnetic force B comprises of two forces emanating from there configured magnetized atoms:

(1) The exposed inner electron (-e)basednegative magnetic (north) pole and

(2) The exposed proton ( $+e$ ) based positive magnetic (south) pole.

These non-moving negative and positive charges at the two ends of a magnet result in a negative magnetic (north) pole with a negative torque $(-\boldsymbol{\tau})$ and a positive magnetic (south) pole of the magnet with an opposing positive torque $(+\boldsymbol{\tau})$. The torque $(\boldsymbol{\tau})$ could be vice versa depending on how you look at it.

\section{Calculating the Tesla unit with the help of Gill's electronic theory of magnetism (1964)}

If we have a force of one newton between the two magnetic poles when they are one meter apart, then we call it a force of one Tesla.

Applying Gill's electronic theory of magnetism (1964), the force of attraction between the two poles of a magnet, $-q$ coulombs at negative magnetic pole $\mathrm{N}$ and $+q$ coulombs at positive magnetic pole Scan be calculated by applyingCoulomb's law (1784).

$F=\frac{k(-q)(+q)}{d^{2}}$ newtons $=-\frac{k q^{2}}{d^{2}}$ newtons.

$d$ is the distance between the two magnetic poles in meters.

Coulomb's

constant $\boldsymbol{k}=\mathbf{8 . 9 9} \times \mathbf{1 0}^{9} \mathbf{N m}^{2} \boldsymbol{C}^{-2}$ where $\boldsymbol{N}$ is for newtons, $\boldsymbol{m}$ is in meters and $\boldsymbol{C}$ is for coulombs.

If $\boldsymbol{F}=\mathbf{- 1}$ newton and $\boldsymbol{d}=\mathbf{1}$ meter, then we have one Tesla unit between the two magnetic poles. Substituting these values, the above equation becomes

$F=-1$ newton $=-\frac{k q^{2}}{1^{2}}=-k q^{2}$ or

$q^{2}=\frac{1}{k}$ or

$q=\frac{1}{\sqrt{k}}$ coulombs

The N-pole has an exposed negative charge $-\boldsymbol{q}=\boldsymbol{n}(-\boldsymbol{e})$ coulombs where $\boldsymbol{n}$ is the number of exposed inner electrons at magnetization.

The S-pole has an exposed positive magnetic charge $+\boldsymbol{q}=\boldsymbol{n}(+\boldsymbol{e})$ coulombs where $\boldsymbol{n}$ is the number of exposed protons at the S-pole.

Then, the above equation becomes

$\boldsymbol{F}=\frac{\boldsymbol{k}(-\boldsymbol{n} \boldsymbol{e})(+\boldsymbol{n} \boldsymbol{e})}{\boldsymbol{d}^{2}}$ newtons $=-\frac{\boldsymbol{k} \boldsymbol{n}^{2} \boldsymbol{e}^{2}}{\boldsymbol{d}^{2}}$ newtons or

$\boldsymbol{n}^{2}=-\frac{F d^{2}}{k e^{2}}$ or in units it is $\frac{N m^{2}}{\left(N m^{2} C^{-2}\right) C^{2}}$.

$n^{2}=\frac{1}{k e^{2}}$ or

$n=\frac{1}{\sqrt{k} \cdot e}=(\sqrt{k} \cdot e)^{-1}$.

$1 e \approx 1.6 \times 10^{-19} \mathrm{C} ;$ Coulombs constant $\boldsymbol{k}=\mathbf{8 . 9 9} \times 10^{9} \mathrm{Nm}^{2} \mathrm{C}^{-2}$. Applying these, we get

$n=\frac{1}{\sqrt{k} \cdot e}=\frac{1}{\sqrt{\left(8.99 \times 10^{9}\right)} \cdot\left(1.6 \times 10^{-19}\right)}=6.591741555135 \times 10^{13}=6.6 \times 10^{13}$

Tesla: If we have a negative magnetic (north) pole with $6.6 \times 10^{13}$ exposed inner electrons and at one meter, we have a south magnetic pole with $6.6 \times 10^{13}$ exposed protons, then the magnetic force of attraction between the two magnetic poles is one Tesla and we could say that each magnetic pole is manifesting a force equal to one Tesla 
From the above discussion, we see that with the application of Gill's electronic theory of magnetism (1964), any Tesla value can be converted to coulombs or vice versa.

The positively charged protons $(+e)$ and the negatively charged electrons $(-e)$ are the two forces responsible for both magnetism and electrical current and this concept will be used to make a more economical 'Bullet Train'.

We will now explain with the application of Gill's electronic theory of magnetism (1964), how a positioning of an L-shaped electro-magnet with its negative magnetic pole at the train wheel level and its positive magnetic pole on the front of the train leads to a levitated 'Bullet Train'.

First, a brief discussion of the behavior between magnetic and electrical forces

\section{Magnetic compass atop a direct electric current carrying wire}

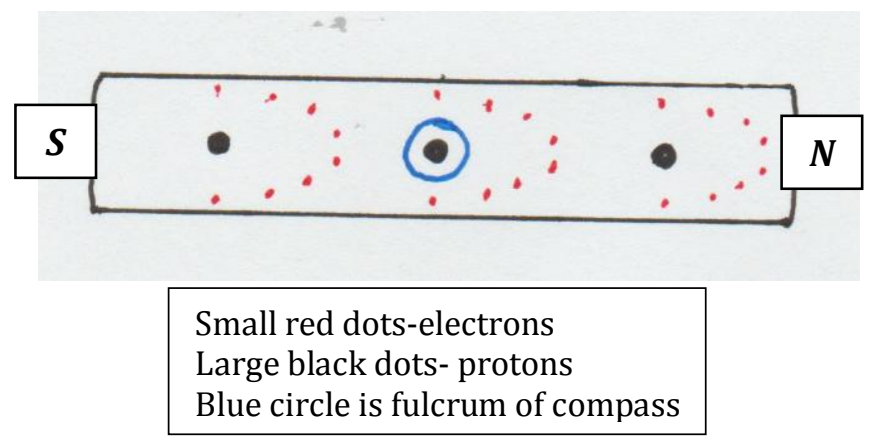

Figure3 (a). Magnetic Compass

Figure3a shows a magnetic compass according to Gill's electronic theory of magnetism (1964), pointing in the North-South direction under the magnetic influence of the Earth's magnetic field. $\boldsymbol{N}$ is the electron $(-e)$ dependent negative magnetic (north) pole and $\boldsymbol{S}$ is the proton $(+e)$ dependent positive magnetic (south) pole.

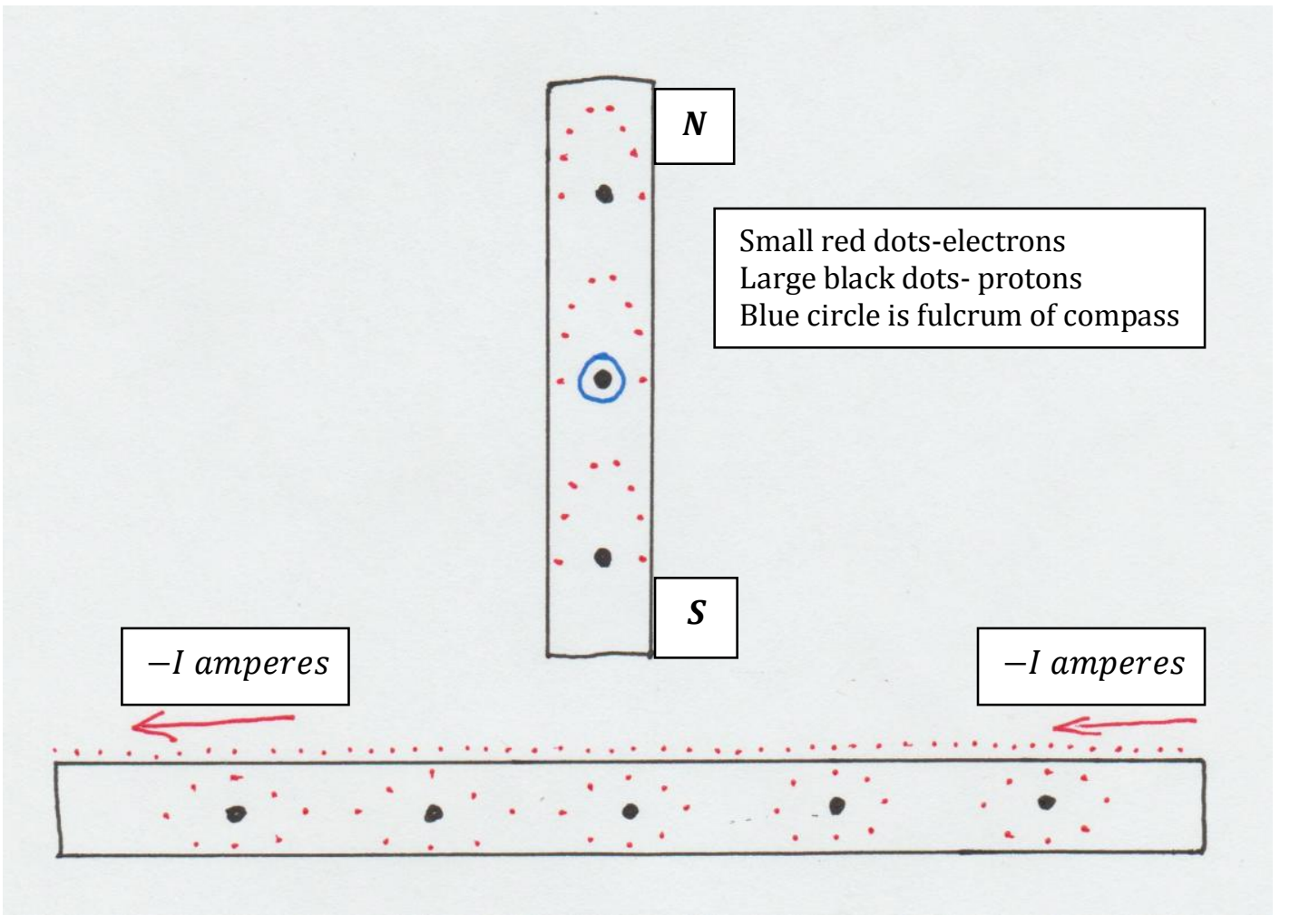

Figure3 (b). The magnetic compass is placed atop an electric wire

In Figure3b, the magnetic compass is placed atop an electric wire which has the negative free valence electrons $(-e)$ flowing on its surface as a continuous negative direct electric current. The proton $(+e)$ dependent positive magnetic pole $\boldsymbol{S}$ is attracted towards the surface electrons $(-e)$ of the direct electric current carrying wire and the electron $(-e)$ dependent negative magnetic pole $\mathbf{N}$ is repelled away from the current carrying wire. 


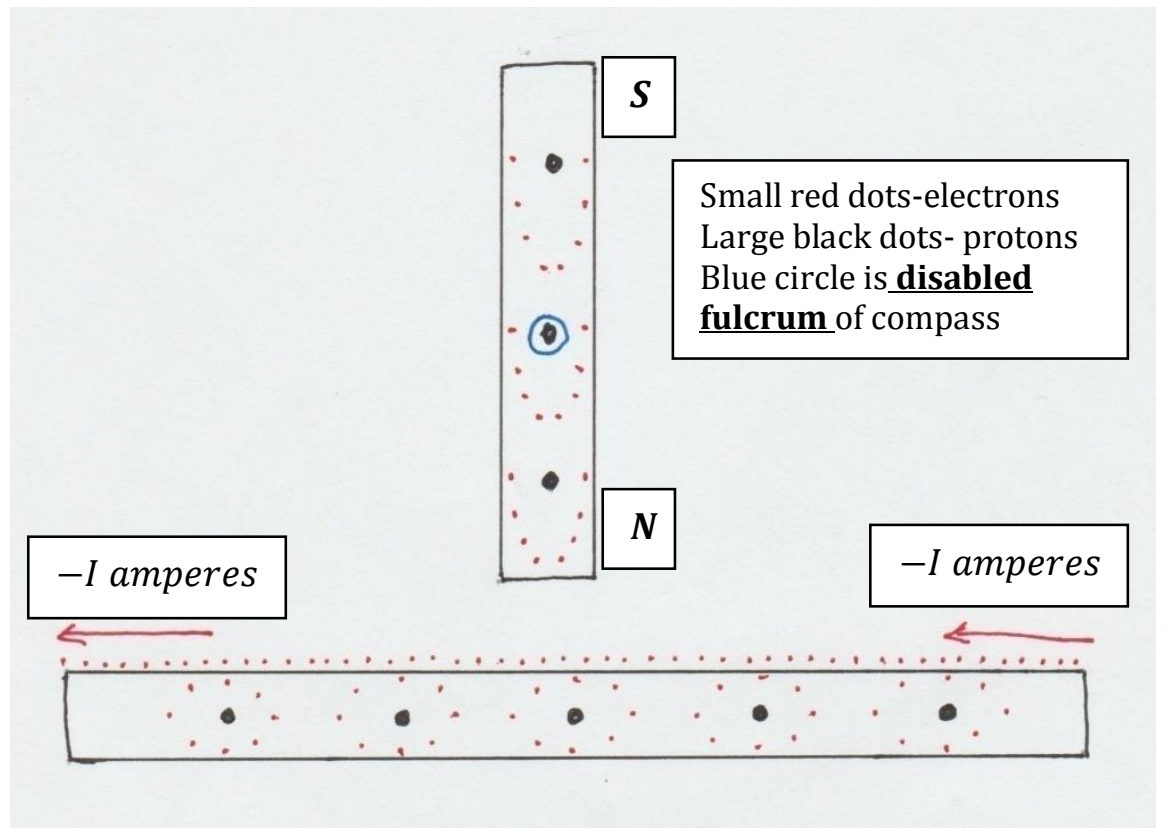

Figure3 (c). Disabled fulcrum of a magnetic compass

Figure $3 c$ shows a disabled fulcrum of a magnetic compass with its electron $(-e)$ dependent negative magnetic pole $\boldsymbol{N}$ forcibly pointed at the track which has an electron $(-e)$ flow on its surface as a continuous negative electron based direct electric current. This will cause repulsion between the electrons $(-e)$ flowing on the conducting strip on the surface of the rail track and the electron $(-e)$ dependent negative magnetic pole $\boldsymbol{N}$ on its surface to cause levitation of the magnet.

We will now proceed with some line diagrams of the 'Bullet Train' followed by calculations for levitation and linear motor function.

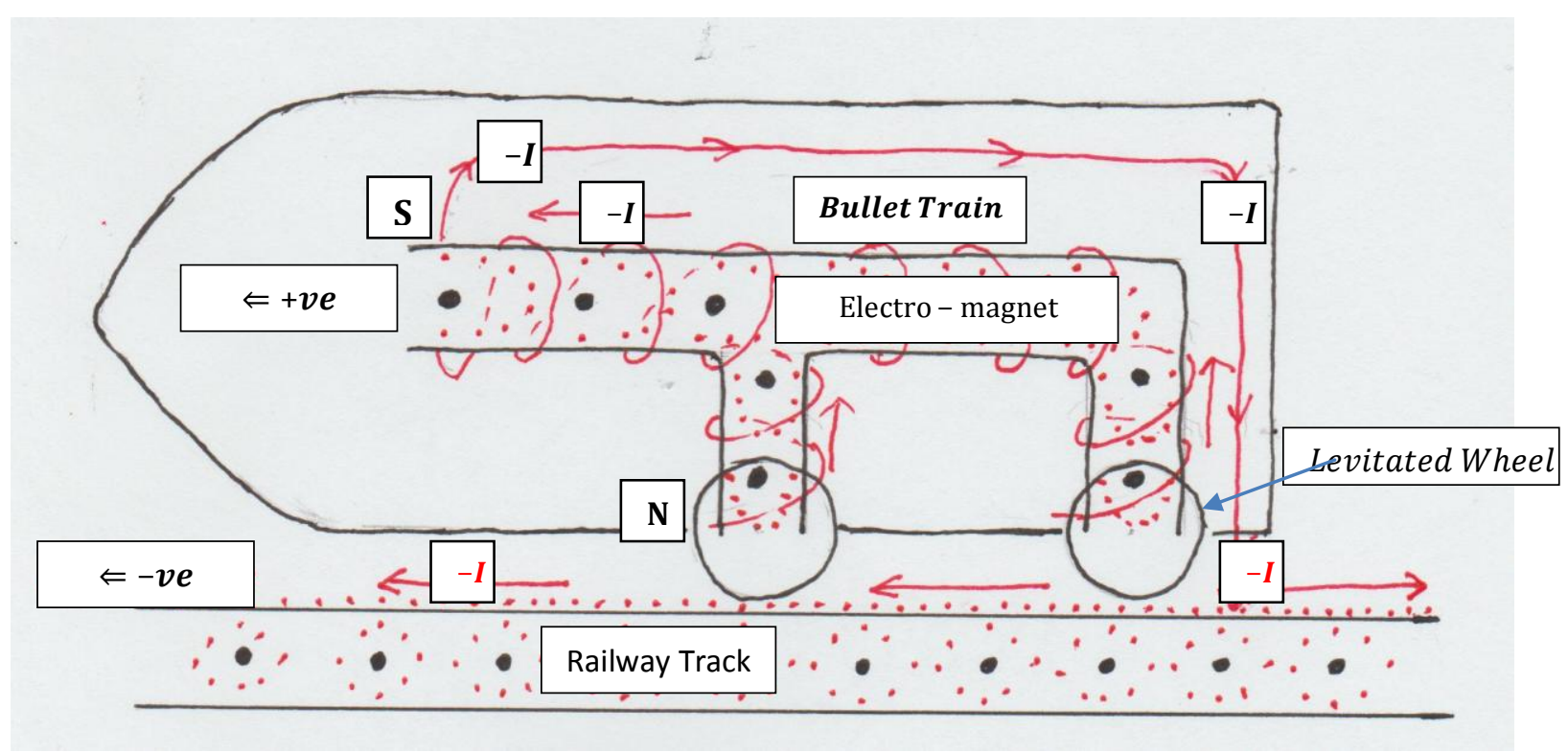

Figure4. The lateral view of the levitated 'Bullet Train' with Gill's electronic theory of Magnetism

As shown by arrows, the same electric current -I used to make the electro-magnet may be diverted towards the rear end of the 'Bullet Train' onto the conducting strip on the rail track.

$\Leftarrow+\boldsymbol{v} \boldsymbol{e}$ sign indicates the positive energy from the exposed proton dependent positive or south magnetic pole $\boldsymbol{S}$ of the electro-magnet which acts as a linear motor and is pulled by the negative energy as it flows along the railway track as a direct electric current.

$\Leftarrow-\boldsymbol{v e}$ sign indicates the negative energy from the negative electron based direct electric current on the surface of the Railway Track which drags the positive magnetic pole $\boldsymbol{S}$ and the train with it. This same negative energy is repelled by the negative magnetic pole $\boldsymbol{N}$ at the train wheel level to cause repulsive levitation. 
In a simplified manner, an L-shaped electro-magnet will be used to address the levitation of the train and its linear motor with the help of its negative and positive magnetic poles:

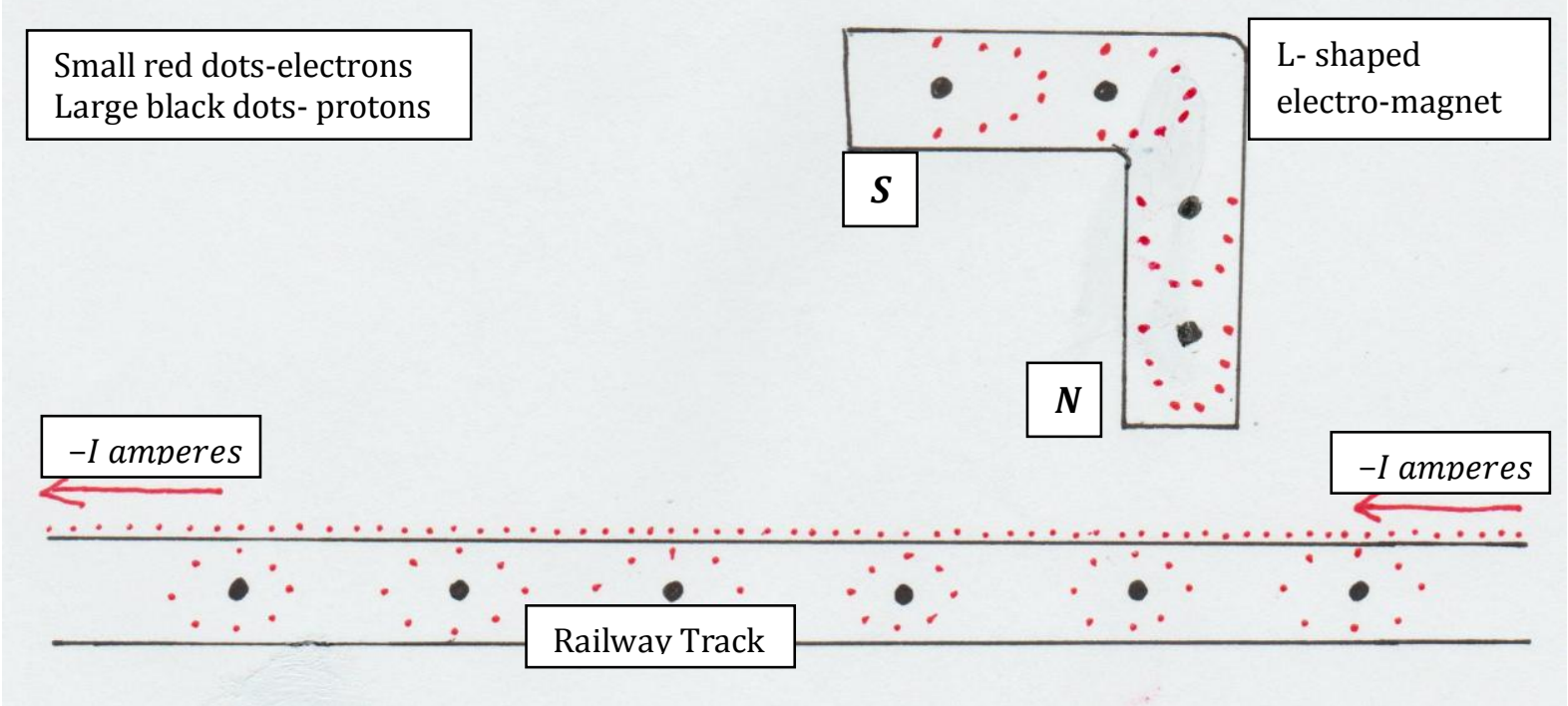

Figure5. Lateral view of a Bullet Train

In Figure5, we are looking at a railway track with electron $(-e)$ based direct electric current (-I amperes) flowing on it with the superficial electrons flowing in the direction of the red arrow. Applying Gill's electronic theory of magnetism to the same, an L-shaped electro-magnet is placed atop the track with its inner electron $(-e)$ based negative magnetic pole $(\boldsymbol{N})$ close to the surface of the track under the train and its exposed proton $(+e)$ based positive magnetic pole $(\boldsymbol{S})$ at a higher level in front of the train on the railway track as shown.

We will deal with the two ends of the L-shaped electro-magnet separately.

Part 1 for levitation of the train by repulsion between the inner electron $(-e)$ dependent negative magnetic pole $(\boldsymbol{N})$ and the electrons $(-e)$ flowing on the Railway Track as a direct negative electric current.

Part 2 for linear motor function of the levitated train along the railway track by the attraction between the exposed proton $(+e)$ dependent positive magnetic pole $(\boldsymbol{S})$ on the front of the train and the electrons $(-e)$ flowing on the Railway Track away from the train as a direct electric current.

Part 1: Applying Coulomb's law (1784), the force of repulsion between the inner electron (-e) dependent negative magnetic pole $(N)$ under the train wheel and the electron $(-e)$ based direct electric current on the railway track is calculated as follows:

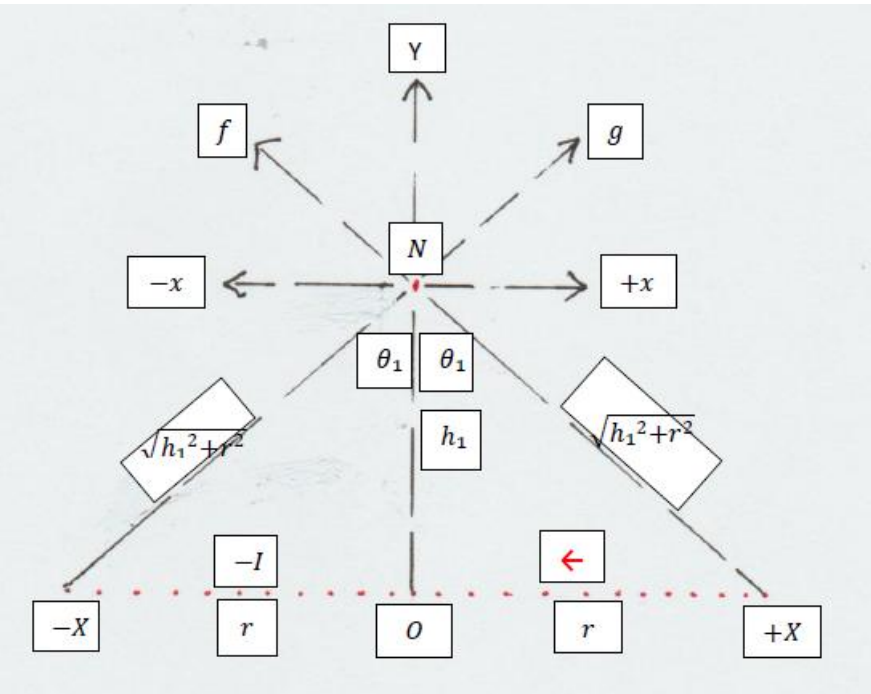

Figure6 (a). Red dot at Nis a non-moving negative electron(-e) dependent charge of the negative magnetic pole under the train wheel.

Red dots from $+X$ to $-X$ are electrons flowing a sa direct current - Ialong the railway track. 
In Figure 6a, $N O$ is a small perpendicular $(\perp)$ height of $h_{1}$ meters (actually a few mille-meters) between the electron dependent negative magnetic pole $(\boldsymbol{N})$ of the electromagnet at the train wheel and the electron based direct electric current in the conducting strip on the surface of the Railway Track.

$-X O=X O=r$ Meters

As $h_{1} \ll-X O$ or $X O$ so $r=\infty$ (infinity)

At point $-X$ we have a charge $\boldsymbol{t}(-\boldsymbol{e})=-\boldsymbol{t} \boldsymbol{e}$ coulombs due to the direct electric current flowing on the railway track where $t$ is the number of flowing electrons at the point- $X$.

At point $\boldsymbol{N}$ we have a non-moving inner electron dependent negative magnetic pole with a negative charge $\boldsymbol{n}(-\boldsymbol{e})=-\boldsymbol{n} \boldsymbol{e}$ coulombs where $\boldsymbol{n}$ is the number of exposed electrons of the negative magnetic pole $(\boldsymbol{N})$.

Using Pythagoras theorem, $-X N=+X N=\sqrt{\boldsymbol{h}_{1}^{2}+\boldsymbol{r}^{2}}$ meters.

Applying Coulomb's law, the force of repulsion between - te at point $-X$ and $-\boldsymbol{n} \boldsymbol{e}$ at point $N$ is a

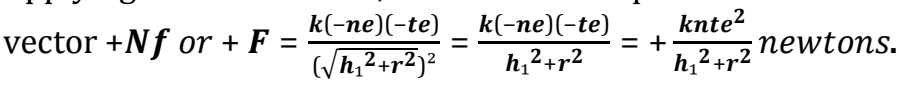

Electron has a negative charge in Coulombs: $-\boldsymbol{e}=-\mathbf{1 . 6} \times \mathbf{1 0}^{-\mathbf{1 9}} \mathrm{C}$

Proton has a positive charge in Coulombs: $+\boldsymbol{e}=-\mathbf{1 . 6} \times \mathbf{1 0}{ }^{-19} \mathrm{C}$.

$\boldsymbol{k}$ is Coulomb's constant: $\boldsymbol{k}=\mathbf{8 . 9 9} \times \mathbf{1 0}^{9} \mathrm{Nm}^{2} \mathrm{C}^{-2}$.

+ sign denotes repulsion and -sign denotes attraction.

The upward vector component $+\boldsymbol{N} \boldsymbol{Y}$ of $+\boldsymbol{N} \boldsymbol{f}$ or force of repulsion/levitation between point $-X$ and point $\boldsymbol{N}$ is

$$
+N Y=F \cos \theta_{1}=\frac{k(-n e)(-t e)}{h_{1}^{2}+r^{2}} \cdot \frac{h_{1}}{\sqrt{h_{1}^{2}+r^{2}}}=+\frac{k n t e^{2} h_{1}}{\left(h_{1}^{2}+r^{2}\right)^{3 / 2}}
$$

The total $N Y$ vector from point $-X$ to point $O$ by adding all the points at a small distance $\Delta \boldsymbol{r}$ from each other over the distance $r=\infty$ on integration is

Total $\boldsymbol{F} \cos \boldsymbol{\theta}_{1}$ from $-\boldsymbol{X}$ to $\boldsymbol{O}=\int_{0}^{\infty}+\frac{\boldsymbol{k n t e ^ { 2 }} \boldsymbol{h}_{1} \boldsymbol{r} \Delta \boldsymbol{r}}{\left(\boldsymbol{h}_{1}{ }^{2} \boldsymbol{r}^{2}\right)^{3 / 2}}=+\boldsymbol{k n t e} \boldsymbol{e}^{2} \boldsymbol{h}_{1} \int_{0}^{\infty} \frac{r \Delta \boldsymbol{r}}{\left(\boldsymbol{h}_{1}{ }^{2}+\boldsymbol{r}^{2}\right)^{3 / 2}}$.

Doing a $\boldsymbol{u}$ substitution where $\boldsymbol{u}=\boldsymbol{h}_{1}{ }^{2}+\boldsymbol{r}^{2}$, then $\Delta \boldsymbol{u}=\mathbf{2} \boldsymbol{r} \boldsymbol{r}$ as $\boldsymbol{h}_{1}$ is a constant, and we have

$$
\text { Total } \boldsymbol{F} \cos \theta_{1} \text { from }-X \text { to } O=+\frac{k n t e^{2} h_{1}}{2} \int_{0}^{\infty} \frac{\Delta u}{(u)^{3 / 2}}=+\frac{k n t e^{2} h_{1}}{2} \int_{0}^{\infty}(u)^{-3 / 2} \Delta u
$$

Integrating the above by taking the anti-derivative of $\boldsymbol{u}^{-\frac{3}{2}}$ which is $-\mathbf{2} \boldsymbol{u}^{-\frac{1}{2}}$ and we have

$$
\text { Total NY from -X to } O=+\frac{k n t e^{2} h_{1}}{2}\left[-2 u^{-\frac{1}{2}}\right]_{0}^{\infty}=+k n t e^{2} h_{1}\left[-u^{-\frac{1}{2}}\right]_{0}^{\infty}=+k n t e^{2} h_{1}\left[-\left.\frac{1}{u^{\frac{1}{2}}}\right|_{0} ^{\infty}\right.
$$

Re-substituting $\boldsymbol{u}=\boldsymbol{h}_{1}{ }^{2}+\boldsymbol{r}^{2}$, we have

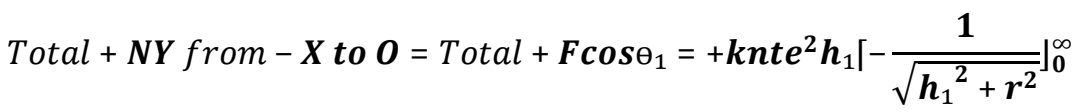

As $\boldsymbol{r}$ is from $\mathbf{0}$ to $\infty$ (infinity) and $\boldsymbol{h}_{1}$ is a constant, on integration we have

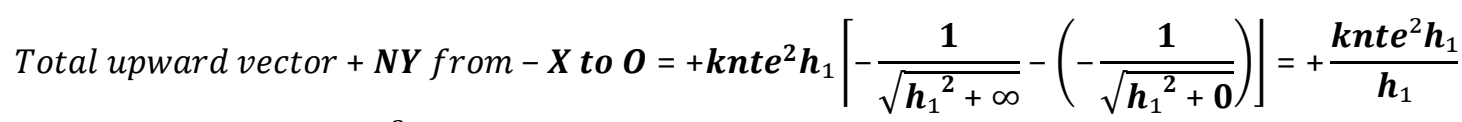

$$
\begin{aligned}
& =+\boldsymbol{k n t} \boldsymbol{e}^{2} \text { newtons } .
\end{aligned}
$$

Using same calculations from $+\boldsymbol{X}$ to $\boldsymbol{O}$,

$$
\text { Total upward vector }+\mathbf{N Y} \text { from }+\boldsymbol{X} \text { to } \mathbf{0}=+\boldsymbol{k n t} \boldsymbol{e}^{2} \text { newtons. }
$$

Figure 6a shows that the lateral vectors from the two sides $+\boldsymbol{x} \boldsymbol{N}$ and $-\boldsymbol{x} \boldsymbol{N}$ cancel each other and we are left with the upward vector force $+N \boldsymbol{Y}$ only which is the upward force of repulsion or levitation of the wheel above the Railway Track. 


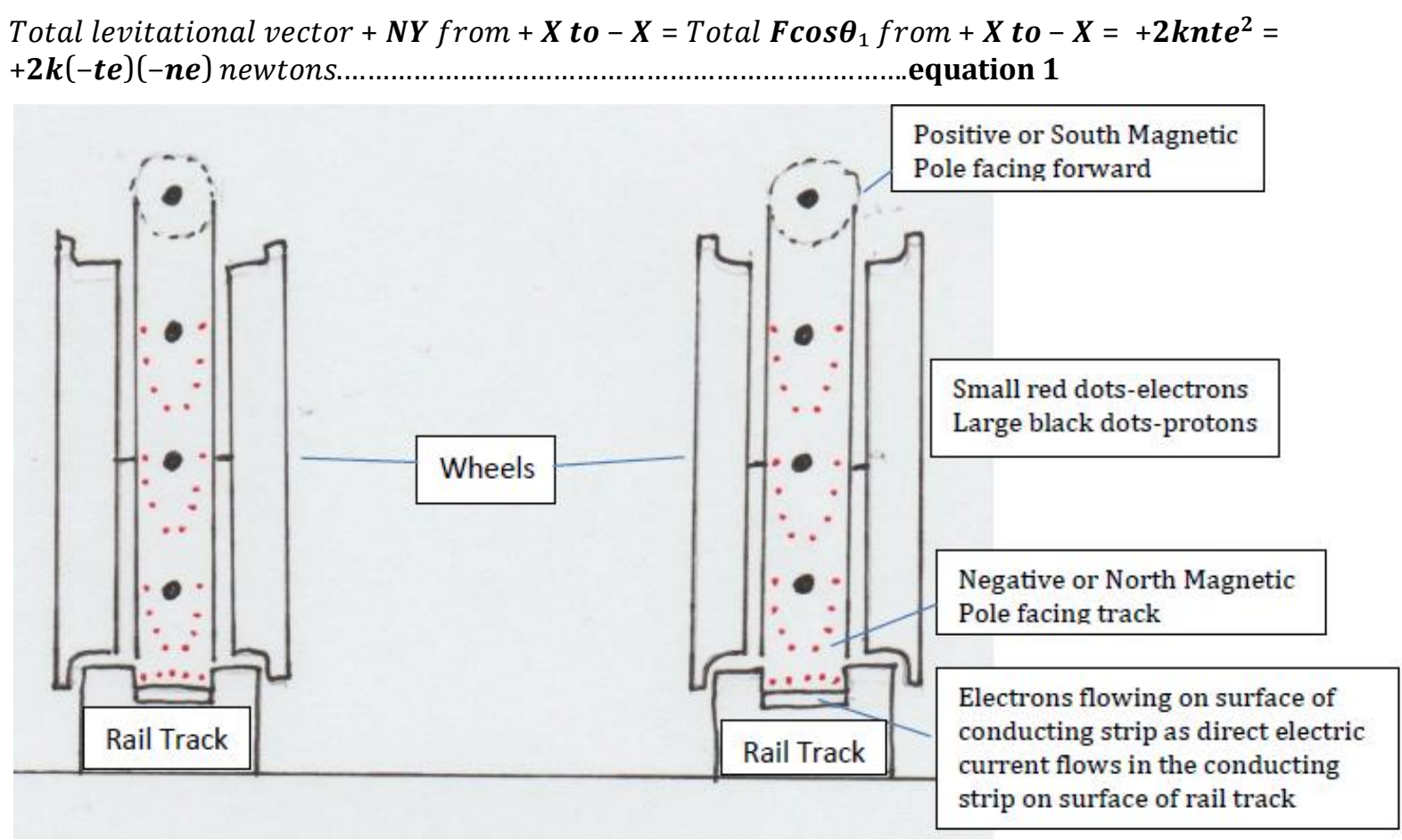

Figure6 (b). Frontal View of Bullet Train with its two tracks

Figure $6 \mathrm{~b}$ shows levitation of the wheels of a 'Bullet Train" by repulsion between the negative electromagnetic pole and the direct negative electric current on the rail track.

As-ne at point $\boldsymbol{N}$ is measured in teslas, we have to do the following calculations for it to be represented in coulombs.

Total upward levitation force $\mathbf{N Y}$ is directly proportional to the negative magnetic force in teslas at $(\boldsymbol{N})=-\boldsymbol{n} \boldsymbol{e}$ coulombswhere $\boldsymbol{n}$ is a number and- $\boldsymbol{e}=-\mathbf{1 . 6} \times \mathbf{1 0}{ }^{-19}$ coulombs.

According to Gill's electronic theory of magnetism (1964), If we have a negative magnetic pole $\boldsymbol{N}$ with $6.6 \times 10^{13}$ exposed inner electrons and at one meter, we have a positive magnetic pole $S$ with $6.6 \times 10^{13}$ exposed protons, then the magnetic force of attraction between the two magnetic poles is one newton and each of those two magnetic poles manifest a force in coulombs to generate one Tesla.

1 tesla $=6.6 \times 10^{13}$ e coulombs Where1e $=1.6 \times 10^{-19}$ coulombs

For example 1 milli - tesla $=6.6 \times \frac{10^{13}}{10^{3}}$ e coulombs $=6.6 \times 10^{10} e$ coulombs

Knowing the tesla value of the negative magnetic pole, we can apply the above to have the - ne value as a non-moving negative charge in coulombs. Thus, Tesla force from the negative magnetic pole can be converted to coulombs or vice versa.

Total levitation force $\boldsymbol{N Y}$ is also directly proportional to the moving electrical charge on the rail track represented as apoint charge $=-\boldsymbol{t} \boldsymbol{e}$ coulombs. Where $\boldsymbol{t}$ is the number of moving electrons $(-\boldsymbol{e})$ at that point and $-\boldsymbol{e}=\mathbf{1 . 6} \times \mathbf{1 0}^{-19}$ coulombs.

Knowing the negative direct electric current is-I amperes and asamperes $=\frac{\text { coulombs }}{\text { second }}$, we can have the point charge-te in coulombs and-te $=-\boldsymbol{I}$ coulombs.

Thus, the train has been levitated with the help of equation 1 and this removes the friction between the train wheels and the railway track. Please note that we aim for a levitation of less than 5 milli-meters and electrical engineers will titrate the values of $\boldsymbol{- n} \boldsymbol{e}$ and-te to get the desired levitation and it will be a small percentage of overall energy consumption.

The levitation is because of the much more economical repulsion between the negative magnetic pole $\mathbf{N}$ and the electrons flowing as a direct electric current on the Rail Track. The author has not used two or more sets of magnets for levitating the train.

Part 2: The linear motor in front of the train will function because of the force of attraction between the exposed proton $(+e)$ dependant positive magnetic pole at point $S$ and the direct electric current-I amperes flowing away as electrons (-e) along the railway track. 


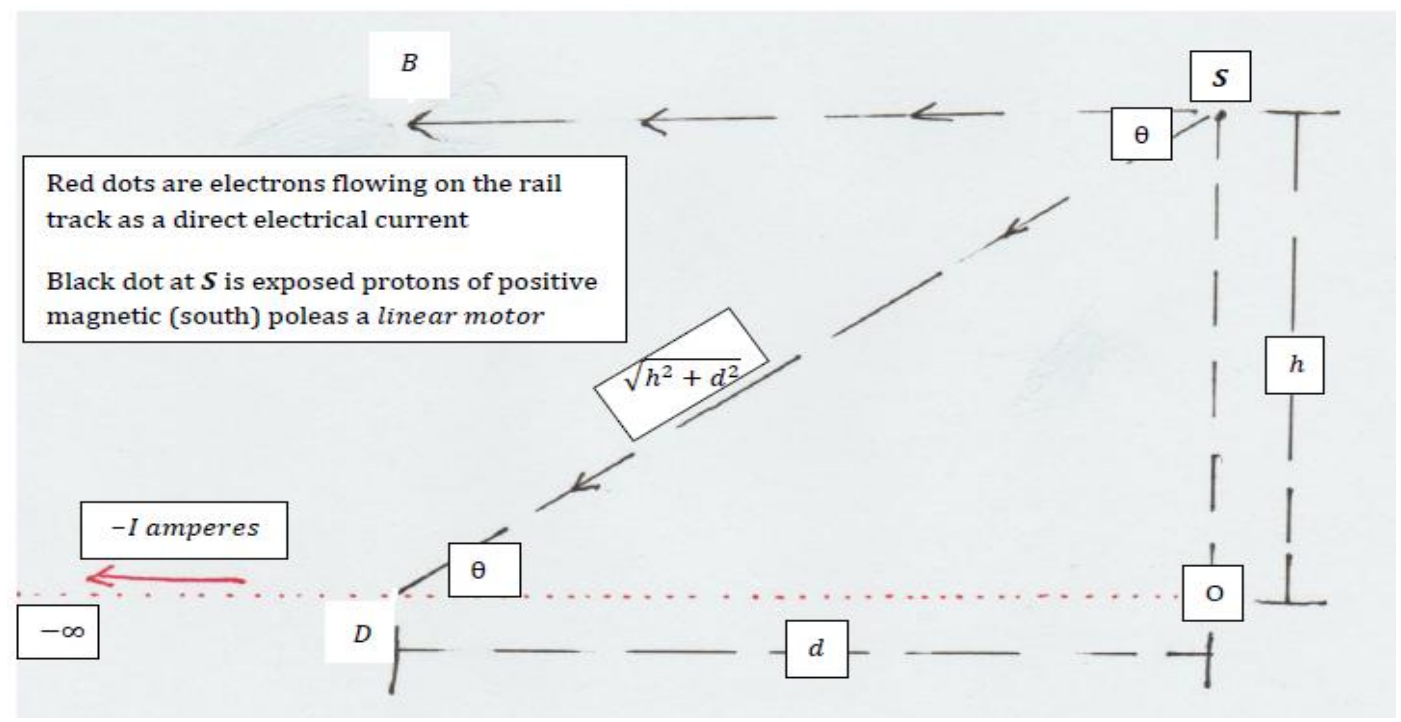

Figure7 (a). The linear motor in front of the train

In Figure 7a, point $\boldsymbol{S}$ is the positive magnetic (south) pole in front of the train at a significant height $\boldsymbol{h}$ (about one meter)above the rail track with its exposed proton dependent positive charge of $+\boldsymbol{q}=\boldsymbol{n}(+\boldsymbol{e})=+\boldsymbol{n} \boldsymbol{e}=\boldsymbol{+} \boldsymbol{n}\left(\mathbf{1 . 6} \times \mathbf{1 0}^{-19}\right)$ coulombs as $\mathbf{1} \boldsymbol{e}=\mathbf{1 . 6} \times \mathbf{1 0}^{-19}$ coulombs where $\boldsymbol{n}$ is the number of exposed protons at point $\boldsymbol{S}$ and $+\boldsymbol{e}$ is the charge of a proton. (Please note that this positive non-moving charge at point $\boldsymbol{S}$ could be made a useful multiple of $+\boldsymbol{n} \boldsymbol{e}$ by adding the electromagnetic forces from under all the wheels to form a larger $\boldsymbol{S}$ in front of the train.)

$O D$ is part of one of the two railway tracks (unless one wants to do the mono-rail track system) with a direct electric current $-I$ amperes flowing from $O \rightarrow D \rightarrow-\infty$ (minus infinity) and the outer loose valence electrons are manifesting as a negative force flowing away from the train on the rail track as a direct electric current.

At point $D$, we have a point charge with $\boldsymbol{t}$ number of flowing electrons each with a charge of $-\boldsymbol{e}$ and the total negative manifest charge at point $D$ is $-\boldsymbol{q}=\boldsymbol{t}(-\boldsymbol{e})=-\boldsymbol{t} \boldsymbol{e}$ coulombs as $-\boldsymbol{e}=-\mathbf{1 . 6} \times \mathbf{1 0}^{-19}$ coulombs.

Let the perpendicular height $(\perp)$ between the rail track at point $O$ and point $S$ be $\boldsymbol{h}$ meters.

The distance $O D$ is $\boldsymbol{d}$ meters.

Please note that $\boldsymbol{h} \gg \boldsymbol{h}_{1}$, as the latter was used in equation $\mathbf{1}$ and is in mille-meters.

Pythagoras theorem shows us that $S D=\sqrt{\boldsymbol{h}^{2}+\boldsymbol{d}^{2}}$.

Using Coulomb's law, the force of attraction between points $S$ and $D$ is

$\boldsymbol{F}=\frac{\boldsymbol{k n}(+\boldsymbol{e}) \boldsymbol{t}(-\boldsymbol{e})}{\left(\sqrt{\boldsymbol{h}^{2}+\boldsymbol{d}^{2}}\right)^{2}}=-\frac{k n t e^{2}}{\boldsymbol{h}^{2}+\boldsymbol{d}^{2}}$ newtons.

From $O \rightarrow D$ over a distance $\boldsymbol{d}$ meters, the electrons that are flowing are at a small distance $\Delta \boldsymbol{d}$ from each other and we have the charge $\boldsymbol{t}(-\boldsymbol{e})$ coulombsat point $D$ that is flowing as a part of-I amperes. The total vector integrated from point $O$ to point $D$ over $\boldsymbol{d}$ meters is

$$
F(\text { total })=\int_{0}^{d}-\frac{k n t e^{2} \cdot d \Delta d}{h^{2}+d^{2}}
$$

Doing $\boldsymbol{u}$ substitution, where $\boldsymbol{u}=\boldsymbol{h}^{2}+\boldsymbol{d}^{2}$, then $\frac{\boldsymbol{d u}}{\Delta \boldsymbol{d}}=\mathbf{2} \boldsymbol{d}$ as $\boldsymbol{h}$ is a constant and $\frac{\boldsymbol{d u}}{\boldsymbol{2}}=\boldsymbol{d} \Delta \boldsymbol{d}$ and the above equation becomes

$F($ total $)=\int_{0}^{d}-\frac{k n t e^{2} \cdot d u}{2 u}=-\frac{k n t e^{2}}{2} \int_{0}^{d} \frac{d u}{u}$.

The derivative of $\log u=\frac{1}{u}$, so anti-derivative of $\frac{1}{u}=\log u$ and on integration we get

$F($ total $)=-\frac{k n t e^{2}}{2}[\log u]_{0}^{d}$ or 
$F($ total $)=-\frac{k n t e^{2}}{2}\left(\log \left(h^{2}+d^{2}\right)-\log h^{2}\right)$

Let us have $\boldsymbol{h}=\mathbf{1}$ metre then $\log h^{2}=\log \mathbf{1}^{2}=\mathbf{0}$.

As $\boldsymbol{d} \gg \boldsymbol{h}$ so $\boldsymbol{h}^{2}+\boldsymbol{d}^{2}=\boldsymbol{d}^{2}$

Thus, the pulling or attraction force $\boldsymbol{F}$ newtons is directly proportional to $\boldsymbol{d}$.

$\boldsymbol{F} \propto \boldsymbol{d}$ and we have

$\boldsymbol{F}(\boldsymbol{t o t a l})=-\frac{k n t e^{2}}{2} \cdot \log d^{2}$ newtons.

As $\log d^{2}=2 \log d$, we get

$F($ total $)=-\frac{k n t e^{2}}{2} \cdot \log d^{2}=-\frac{k n t e^{2}}{2} \cdot 2 \log d=-k n t e^{2} \cdot \log d$ newtons

If $\boldsymbol{d}=(\mathbf{1 0})^{\mathrm{R}}$ metres where $\boldsymbol{R}$ is an exponent, then we have

$\boldsymbol{F}($ total $)$ along $\boldsymbol{S D}=-\boldsymbol{k n t e} \boldsymbol{e}^{2} \cdot \log (\mathbf{1 0})^{\mathrm{R}}=-\boldsymbol{k n t e} \boldsymbol{e}^{2} \cdot \boldsymbol{R} \log (\mathbf{1 0})$ newtons. The minus sign indicates attraction between the positive magnetic pole $\boldsymbol{S}=+\boldsymbol{q}$ coulombs $=\boldsymbol{n}(+\boldsymbol{e})$ coulombs and the point charge $=\boldsymbol{t}(-\boldsymbol{e})=-\boldsymbol{t} \boldsymbol{e}$ coulombs $=-\boldsymbol{I}$ coulombs flowing as a direct electric current of $-\boldsymbol{I}$ amperes on the railway track from $\boldsymbol{O} \rightarrow \boldsymbol{D}$.

The dot-product vector component $\boldsymbol{S B}$ parallel to the rail track of vector $\boldsymbol{S D}$ will be given by multiplying by $\cos \boldsymbol{\theta}$. As $\boldsymbol{d}$ is approaching infinity, $\angle \boldsymbol{B S D}$ or $\boldsymbol{\theta}=\mathbf{0}^{\circ}$ and $\cos \mathbf{0}^{\circ}=\mathbf{1}$.

$\boldsymbol{F}($ total $) \cos \theta=F($ total $) \cos 0^{\circ}=-\boldsymbol{k n t e}^{2} . \boldsymbol{R l o g}(\mathbf{1 0})$ newtons or

$\boldsymbol{F}($ total $) \cos 0^{\circ}=-k_{n t e} \cdot \operatorname{Rlog}(10)$ newtons equation 2

The positive magnetic pole $\boldsymbol{S}$ pointed forward in front of the train functions as a linear motor.

A flexible linear motor on the front of the 'Bullet Train'

If we place a positive electro-magnetic pole ( $\boldsymbol{S}$ ) with a flexible core in front of the train about one meter above the rail track, the direction of this flexible positive electro-magnetic pole (S) can be altered to vary the speed and even the direction of the levitated 'Bullet Train'.

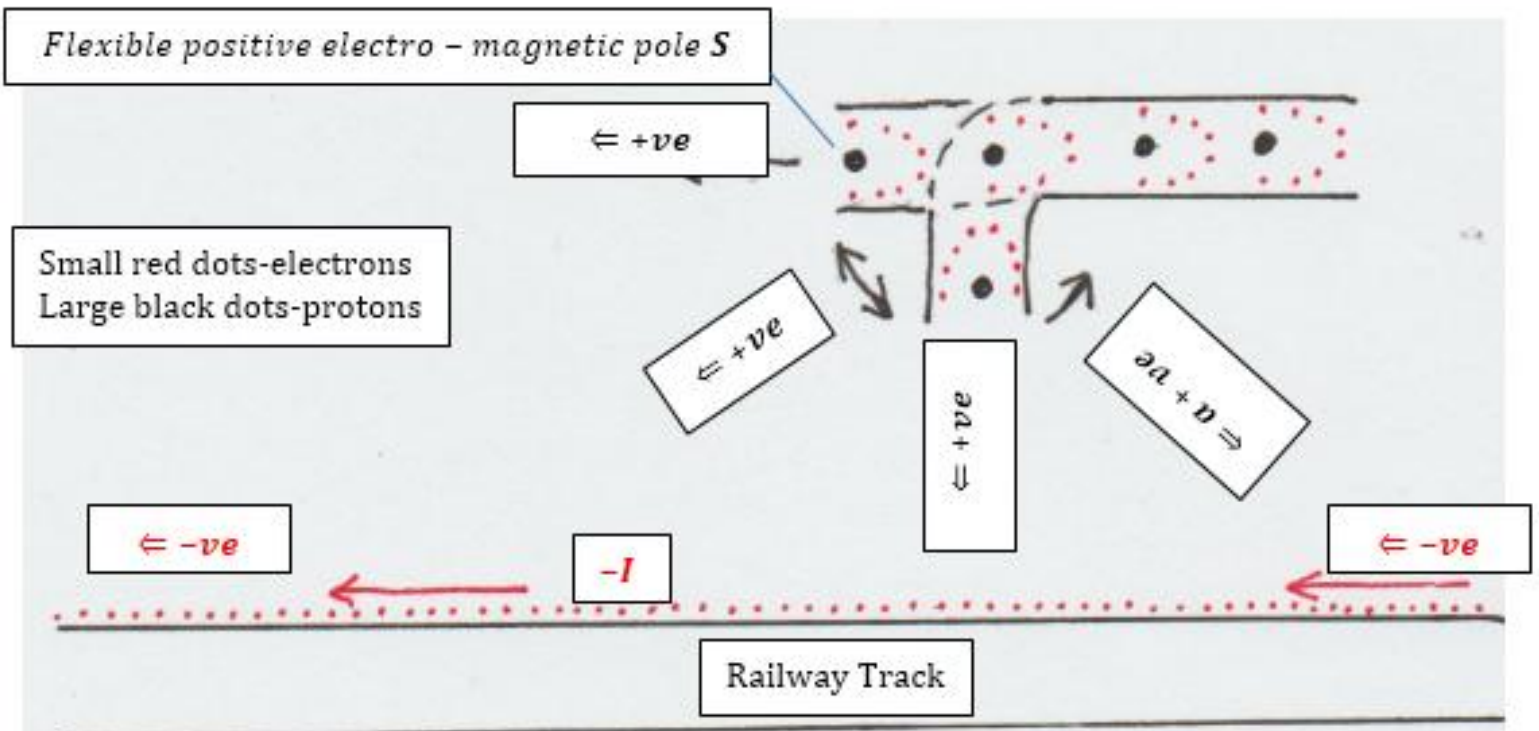

Figure7 (b). Positive electro-magnetic pole $S$ has a flexible core 
Equation 2 shows the total forward attractional force between the train and the rail track as

$\boldsymbol{F}($ total $) \cos \theta=-k n t \boldsymbol{e}^{2} . \boldsymbol{R} \log (\mathbf{1 0}) \cos \theta$ newtons.

When distance $\boldsymbol{d}$ is approaching infinity $(\infty), \boldsymbol{\theta}=\mathbf{0}^{\circ}$, then $\cos \boldsymbol{\theta}=\boldsymbol{\operatorname { c o s }} \mathbf{0}^{\circ}=\mathbf{1}$, then we have maximal attractional force on the levitated train which is

$\boldsymbol{F}($ total $) \cos \mathbf{0}^{\circ}=-\boldsymbol{k n t e}^{2} . \operatorname{Rlog}(10)$ newtons.

If the positive electro-magnetic pole $(\boldsymbol{S})$ is pointed downwards towards and pependicular to the track, then $\boldsymbol{\theta}=\mathbf{9 0}^{\circ}$, and $\cos \boldsymbol{\theta}=\cos \mathbf{9 0 ^ { \circ }}=\mathbf{0}$ and the equation 2 becomes

$\boldsymbol{F}($ total $) \cos 90^{\circ}=-\boldsymbol{k n t e}^{2} . \boldsymbol{R l o g}(\mathbf{1 0})$ newtons $\times 0=0$.

In this situation, the train though levitated will stay stationary.

If the positive electro-magnetic pole $(\boldsymbol{S})$ is pointed further towards the train, then $\boldsymbol{\theta}=\mathbf{1 2 0}^{\circ}$, and $\cos \boldsymbol{\theta}=$ $\cos 120^{\circ}=-\frac{1}{2}$ and the equation 2 becomes

$\boldsymbol{F}(\boldsymbol{t o t a l}) \cos \mathbf{1 2 0}^{\circ}=\left(-\boldsymbol{k n t e}^{2} \cdot \boldsymbol{R l o g}(\mathbf{1 0})\right)\left(-\frac{1}{2}\right)$ newtons $=+\frac{\boldsymbol{k n t e ^ { 2 }} \cdot \boldsymbol{R l o g}(\mathbf{1 0})}{2}$ newtons.

In this situation, the train will be moved backwards or in the opposite direction. This situation could be used for slowing down the levitated train.

In Figure 7b, we have applied Gill's electronic theory of magnetism and we see:

So, if the $\boldsymbol{S}$ end of the electro-magnet is pointed downwards towards the rail track at the start, the train will not move when levitated. As the flexible electro-magnetic end $\boldsymbol{S}$ of the levitated train is slowly pointed or rotated forward from $\mathbf{9 0}^{\circ}$ to $\mathbf{0}^{\circ}, \cos \boldsymbol{\theta}$ will keep on increasing from zero to one and the levitated train will keep on increasing its speed.

Equation 2 with a minus sign indicating attraction serves as an attractional tether between the train and the railway track and pulls the levitated train in the direction of flow of electrons or direct electric current $(-I)$ on the railway track.

The moving direct electric charge $-I \frac{\text { Coulomb's }}{\text { second }}$ or - I amperesin the railway track moves the levitated train from a point $\mathrm{O}$ below the train towards $-\infty$ (minus infinity) along the railway track.

Equation I with the help of the negative magnetic pole $\boldsymbol{N}$ levitates the train.

A very high frequency alternating current synchronized between the electro-magnet and the railway track could produce a result similar to the direct electric current just discussed.

\section{DisCUSSION}

In the Maglev system (referring to the magnetic levitation), the 'Bullet Train' is a fast-moving levitated train transportation system where the levitation and the longitudinal movement is caused by two sets of magnets:

These two sets of magnets arrangement is necessitated by adherence to Maxwell's dipole theory of magnetism (1873).

A: one set of magnets to repel and levitate the train off the tracks and

B: second set of magnets or magnetic effects function as a linear motor to cause the levitated friction-less train to move forward at great speed.

The two known notable types of maglev technology are:

A: Electromagnetic suspension (EMS) in which electronically controlled electromagnets in the train attracts it to a magnetically conductive track.

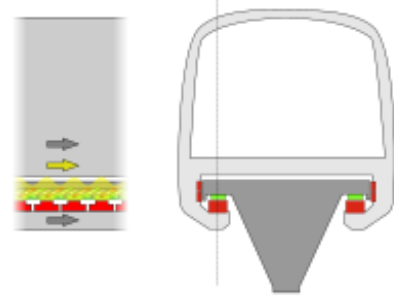


Electromagnetic suspension (EMS) shown above is caused by the electromagnets attached to the train which are oriented toward the steel rail from below to levitate the train. C-shaped arms under the train contain magnets and the rail is situated between the upper and lower edges of the $\mathrm{C}$.

B: Electrodynamic suspension (EDS)

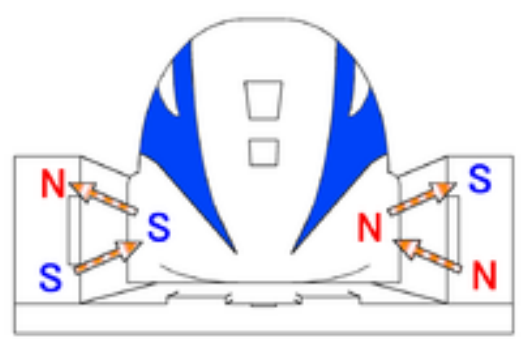

Electrodynamics suspension (EDS) shown above is powered by magnetic fields induced on either side of the vehicle by the passage of the vehicles superconducting magnets.

The negative (north) and positive (south) magnetic poles of the same electro-magnet have been dealt with separately and Gill's electronic theory of magnetism (1964) has been applied.

Part1 deals with the non-moving but manifest negative magnetic charge at $\boldsymbol{N}=-\boldsymbol{q}=-\boldsymbol{n} \boldsymbol{e}$ coulombs of the negative magnetic pole repelled by the free valence electron flow $\boldsymbol{t}(-\boldsymbol{e})=-\boldsymbol{t} \boldsymbol{e} \frac{\text { coulombs }}{\text { second }}=-\boldsymbol{I}$ amperesas direct electric current on the rail track resulting in levitation as in equation 1.

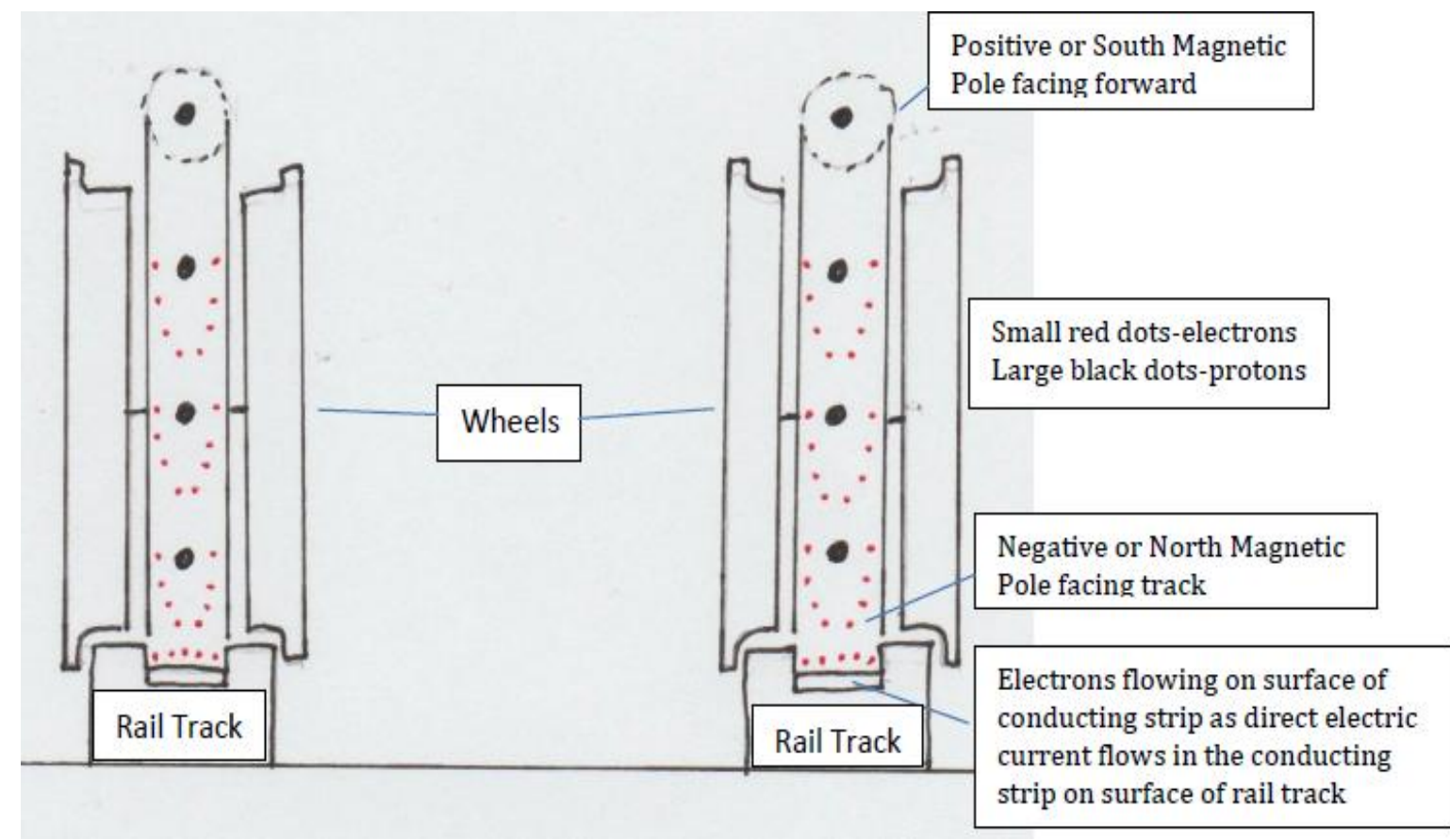

Frontal View of Bullet Train with Gill's electronic theory of magnetism (1964)

Total upward vector $\mathbf{N Y} \uparrow$ from $+X$ to $-X=+2 \boldsymbol{k n t e} \boldsymbol{e}^{2}=\mathbf{2} \boldsymbol{k}(-\boldsymbol{n e})(-\boldsymbol{t e})=+\mathbf{2 k}(\boldsymbol{n e})(\boldsymbol{t e})$ newtons equation 1

Unlike the Magelev system of levitation where two or more sets of magnets are applied, in the above figure we see the levitation is because of repulsion between the negative magnetic pole (-ne coulombs) and the negative electric current (-te $\frac{\text { coulombs }}{\text { second }}=-I$ amperes) flowing on the conducting strip on the Rail Track.

The electron based negative energy of the negative magnetic pole $(N)$ will be repelled by the electron based direct negative electric current on the surface of the rail track and there will be levitation of the wheels which can be titrated to our liking.

At slow speeds, the initial levitation might need time and for this reason, the train must have wheels to support the train until it reaches take-off speed. The entire track must be able to support low and highspeed operation.

Part2 deals with the modified linear motor 


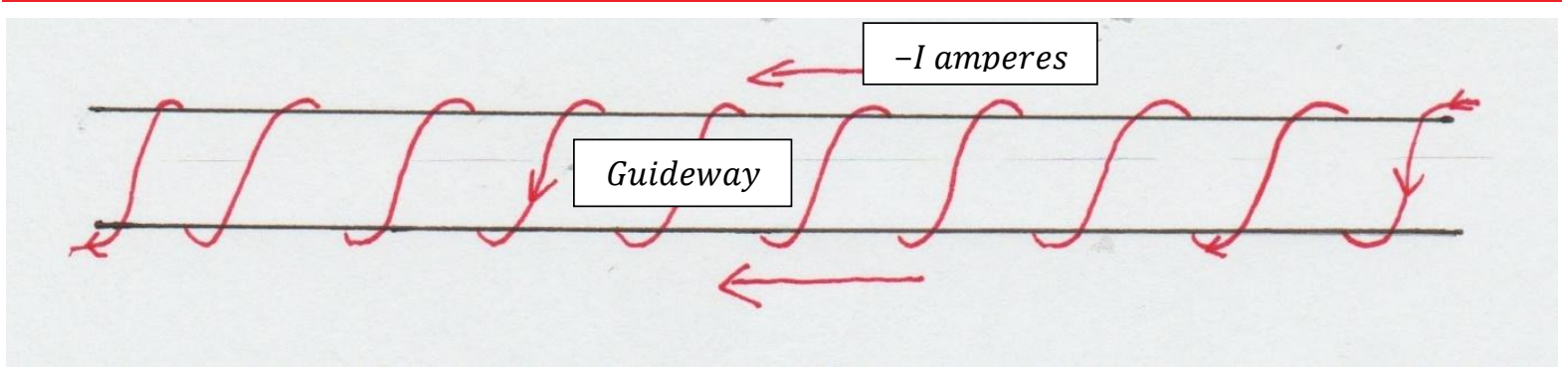

Figure8 (a). Eric Laithwate's (1974) Propulsion coil on Guideway

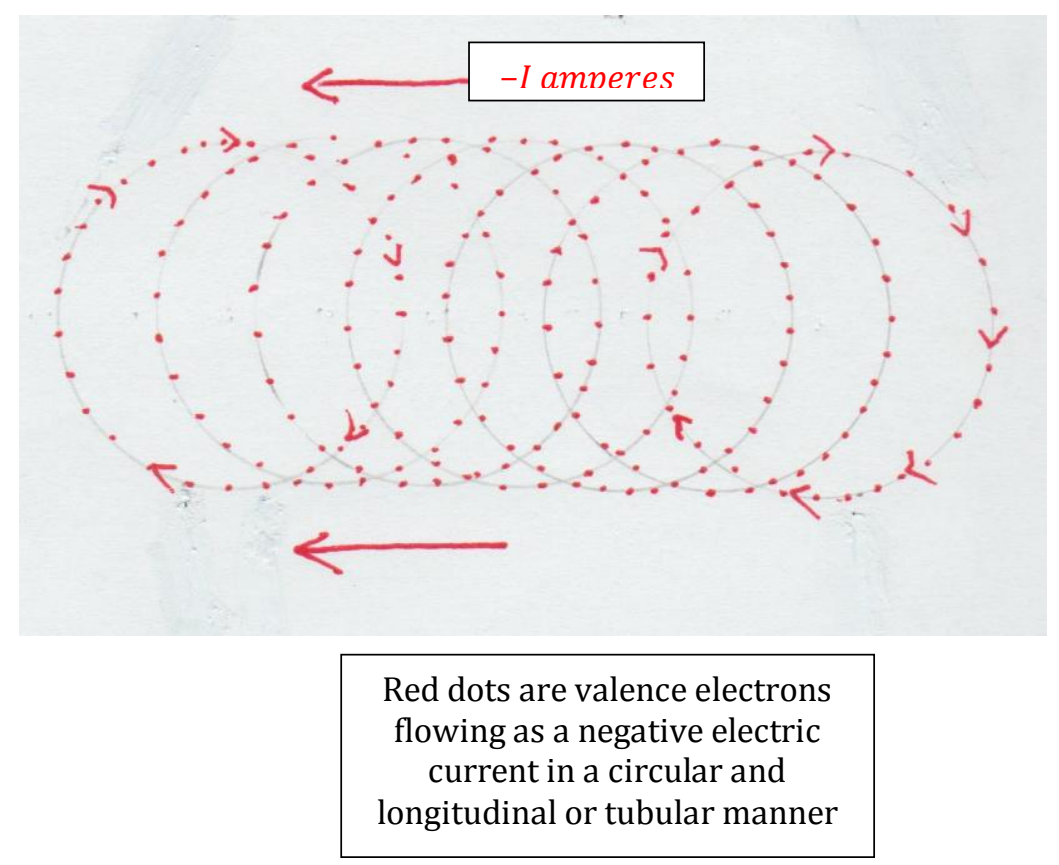

Figure8 (b). Electronic representation of Propulsion coil

Figure $8 \mathrm{a}$ and Figure $8 \mathrm{~b}$ represent the same propulsion coil on the guideway or rail road in front of the 'Bullet train' which has been used by Eric Laithwaite and others since 1974. Propulsion coils on the guide way exert a force on the magnets in the train and make it move forward. This is effectively a linear motor.

In Figure 8a we see a circular flow of electrons which are also flowing longitudinally away from the train as a tubular flow of electrons. This propulsion coil results in attraction between the positive magnetic pole in front of the train and the electrons flowing away from the train in a longitudinal manner. According to Gill's electronic theory of magnetism (1964), the levitated 'Bullet train' needs only the longitudinal tubular flow of electrons to be attracted by the positive magnetic pole $\boldsymbol{S}$ on the front of the train. The torsion caused by the rotation of the electrons in the propulsion coil is not needed. This purely circular flow of electrons causes a torsion which is not needed and this has no role in moving the 'Bullet train forward.

In a simplified manner, an L-shaped electro-magnet will be used to address the levitation of the train and its linear motor with the help of its negative and positive magnetic poles:

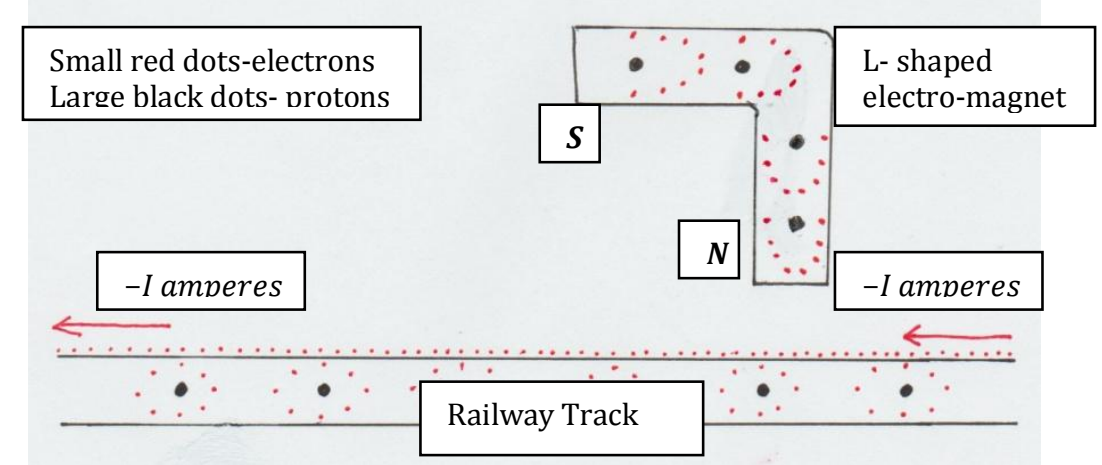

Figure8 (c). Lateral view of a Bullet Train with applied Gill's electronic theory of magnetism 


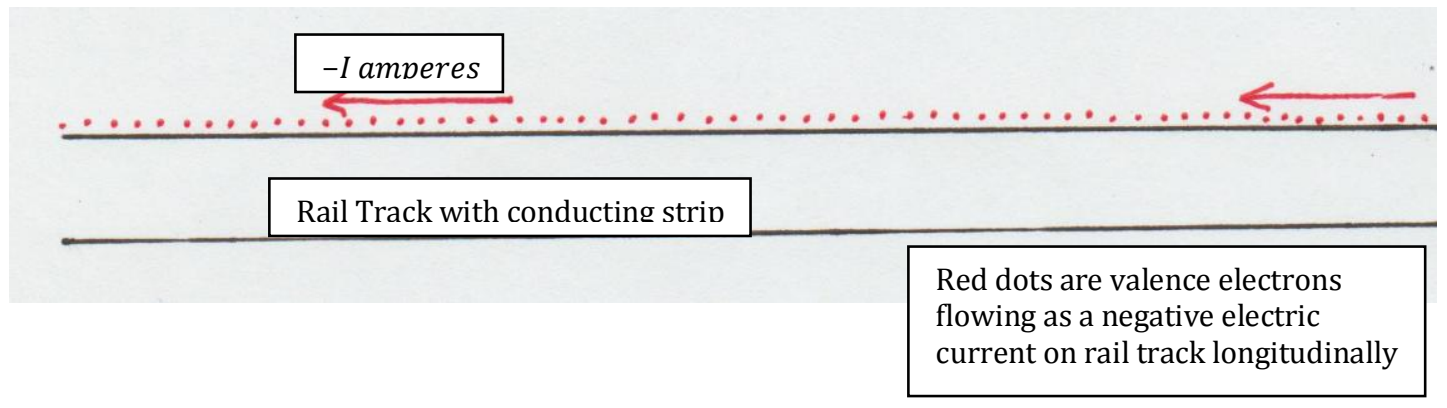

Figure8 (d). Propulsion with conducting strip on rail track

As shown in Figure 8c and Figure 8d, If we use a conducting metal strip on the surface of the rail track, the longitudinal flow of electrons as a direct electric current could be treated as a part of the propulsion coil and will attract the train forward at great speed. Please note that a simple conducting strip costs much less than the propulsion coil.

No propulsion coils are needed in the linear motor system presented with the application of Gill's electronic theory of magnetism (1964). A simple direct negative electric current flowing away from the train in a conducting strip on the rail track or guide way is attracted by the positive magnetic pole in front of the train. As the propulsion coil is a tubular longitudinal flow of electrons, the simple direct negative electric current flowing away from the train on the rail track as a conducting strip could be considered a section of the propulsion coil and behaves similarly except that there is no unnecessary torsion.

We deal with the non-moving but manifest positive magnetic pole charge at $\boldsymbol{S}=+\boldsymbol{q}=+\boldsymbol{n} \boldsymbol{e}$ coulombs of the positive magnetic pole interacting with the free valence electron $(-\boldsymbol{e})$ based charge $\boldsymbol{t}(-\boldsymbol{e})=$ -te coulombs flowing as $-t$ e $\frac{\text { coulombs }}{\text { second }}=-\boldsymbol{I}$ amperes on the rail track resulting in the total forward moving force of the linear motor:

$\boldsymbol{F}($ total $)=-\boldsymbol{k n t e}^{2} \cdot \log (10)^{\mathrm{R}}=\boldsymbol{k}(+\boldsymbol{n e})(-\boldsymbol{t e}) \cdot \boldsymbol{R} \log (10)$ newtons equation 2

Equation 2 shows that the greater the distance $\boldsymbol{d}=\mathbf{1 0}^{\mathrm{R}}$ meters, which is increasing exponentially depending on $(\mathrm{R})$, the greater the speed of the train.

Electrical engineering will titrate the above numbers for best results. The $\boldsymbol{S}$ could be made a significantly increased multiple of $+\boldsymbol{n} \boldsymbol{e}$ coulombs as each wheel will need an electro-magnetic-ne coulombs for repulsion or levitation and all these wheel-based -ne coulomb for sec can be joined to have a much larger multiple of $+\boldsymbol{n} \boldsymbol{e}$ coulombs at the front end as a part of the increased $\boldsymbol{S}$.

The same direct electric current used to cause the electro-magnet could be directed towards the surface of the rail track at the rear end of the train for a direct electric current on the surface of the rail track for economic reasons.

At the start, the direction of $\boldsymbol{S}$ will be downward and after levitation takes place, the $\boldsymbol{S}$ will be slowly pointed forward to make the train gather speed and will be horizontal to the rail track for maximum effect and maximum speed.

The above positive and negative non-moving magnetic charges at the two poles of a magnet as explained by Gill's electronic theory of magnetism (1964) allow dot-product vector assessment and application. The two forces from the negative magnetic pole $\boldsymbol{N}=-\boldsymbol{n}$ e coulombs and positive magnetic pole $\boldsymbol{S}=$ $+\boldsymbol{n}$ e coulombs have been addressed separately and only dot product vectors need to be used in their interaction with the electric current on the railway track. This is not possible with Maxwell's dipole theory of magnetism (1873) which forced Lorentz to come up with the cross-product equations in 1893.

If we apply Gill's electronic theory of magnetism (1964) there is no asymmetry between the magnetic force and the electrical forces as shown in this article. Gill's electronic theory of magnetism (1964)also shows there is no asymmetry issue as it simplifies the explanation of why a moving magnet induces an electric current and vice versa. An asymmetry issue arose due to Maxwell's dipole theory of magnetism (1873) about which Albert Einstein complained in 1905 and tried to explain in the Special Relativity theory in his 1905 article. As there is no asymmetry, there is no need to explain the same.

Although a direct electric current will result in a smoother ride on a "Bullet Train', a very high frequency alternating current synchronized between the electro-magnet and the rail track surface could achieve the same.

Application of Gill's electronic theory of magnetism (1964) in this article facilitates dot-product calculations and equations which explain both levitation and rapid longitudinal movement of a train along 
the rail track with a linear motor and there is no need for the cross-product equations of Lorentz law (1893).

This same negative direct electric current based magnetizing coil for the electromagnet below the train could also be used to pass a direct negative electric current below the train by touching the conducting strip on the rail track at the rear end to cause it to flow in the direction of the train movement. This negative direct electric current on the metal strip on the rail track will attract the positive magnetic pole at the front of the train as discussed in the presentation.

The same electric coil for an electro-magnetic effect could be used to create multiple small north magnetic poles below each wheel of a train could all be combined to result in a large south magnetic pole at the front of the train. This technique offered by the author will be more economical and the forces are easier to calculate with the dot product calculations.

$\boldsymbol{N}$ is the inner electron dependent negative magnetic pole and $\boldsymbol{S}$ is the exposed proton dependent positive magnetic pole and this is explained by a combination of Ampere's right-hand rule (1825) and Gill's electronic theory of magnetism (1964).

Gill's electronic theory of magnetism (1964) leads us down the path of dot-product calculations as shown in this article. Cross-product calculations put forward by Lorentz in 1893 are not needed

\section{CONCLUSION}

Gill's electronic theory of magnetism (1964) explains the basis for a magnet to be addressed as having a negative magnetic pole and a positive magnetic pole. The physics behind a levitated 'Bullet Train' gets simplified. An electric current repelling the negative magnetic pole for levitation and an attraction between the electric current and the positive magnetic pole of the electromagnet for pulling the train forward as a linear motor. Using a magnet repelling another magnet as in Maglev levitation or a magnet being pulled by a magnetic force to move the train forward makes it cumbersome and expensive.

It also supports Gill's electronic theory of magnetism (1964) which states that the magnetic force is a combination of exposed inner electron dependent non-flowing charge $\boldsymbol{n}(-\boldsymbol{e})$ coulombs $=-\boldsymbol{n} \boldsymbol{e}$ coulombs at the negative magnetic pole or $\mathrm{N}$-pole of the magnet and the exposed proton dependent non-flowing charge $\boldsymbol{n}(+\boldsymbol{e})$ coulombs $=+\boldsymbol{n} \boldsymbol{e}$ coulombs at the positive magnetic pole or S-pole of a magnet where $\boldsymbol{n}$ is the number of exposed electrons at the north magnetic pole which is also equal to the number of exposed protons at the south magnetic pole.

The feasibility of the 'Bullet Train' proves Gill's theory of magnetism (1964) to be better than Maxwell's theory of magnetism (1873) and Lorentz's force law (1893) is not needed. Only dot-product vectors are presented for the calculations.

Gill's electronic theory of magnetism (1964) shows that while addressing physics issues, it is better to address a magnet as having a negative magnetic pole and a positive magnetic pole (instead of north and south magnetic poles) while addressing the magnets interaction with the positive and negative electrical forces.

According to Gill's electronic theory of magnetism (1964), the forces from the exposed inner electron (-e) dependant negative (north) magnetic pole and the exposed proton $(+e)$ dependent positive (south) magnetic pole of a magnet on the one hand and the negative $(-e)$ and positive $(+e)$ electrical current forces on the other hand are two sides of the same coin. The positively charged protons and the negatively charged electrons are responsible for both magnetic and electrical forces.

Gill's electronic theory of magnetism (1964) does away with the asymmetry issue raised by A. Einstein in 1905 and Richard Feinmann in 1943. The interaction between the two components of the magnetic force and the electrical force lays to rest the asymmetry issue.

\section{ACKNOWLEDGEMENTS}

I am grateful to physicist Professor Amitabha Mukherjee (former head of department of Physics, Delhi University) for going over this article with me.

Patent

$\underline{53024482} \quad \begin{aligned} & \underline{\text { Provisional }} \\ & \underline{\text { Application for }} \\ & \underline{\text { Patent }}\end{aligned}$
Bullet Train designed with the application of my Gill's electronic theory of magnetism (1964).
$05 / 09 / 2018$ 


\section{REFERENCES}

1. Faraday's iron ring experiment (1831) explained with Gill's electronic theory of magnetism (1964) by author ARJPS 2017

2. A Treatise on Electricity and Magnetism by James Clerk Maxwell (1851).

3. Ampère's circuital law by Andre Marie Ampere (1823)

4. Five Hundred Miles an Hour by Boris Weinberg

5. Magelev system from Wikipedia

6. Coulomb's law by Charles Augustin de Coulomb (1784)

7. Lorentz force by Hendrik Lorentz (1893)

8. ON THE ELECTRODYNAMICS OF MOVING BODIES By A. EINSTEIN June 1905

9. Tesla unit by Nikola Tesla

10. Elementary charge units

Citation: Avtar Singh Gill. "Bullet Train Physics with Gill's Electronic Theory of Magnetism (1964)", American Research Journal of Physics, 4(1):1-17.

Copyright (C) 2018 Avtar Singh Gill, This is an open access article distributed under the Creative Commons Attribution License, which permits unrestricted use, distribution, and reproduction in any medium, provided the original work is properly cited. 